East African Medical Journal Vol. 81 No. 6 June 2004

COMPARISON OF THREE METHODS OF COLLECTING NASAL SPECIMENS FOR RESPIRATORY VIRUS ANALYSIS

M.J. Ngama, Clinical Officer, B., Ouma, HND, Microbiology Senior Laboratory Technician, Kenya Medical Research Insitute, Centre for Geographic Medicine Research (Coast), Kilifi, Kenya, M. E., English, MD, Paeditrician Senior Research Scientist, Kenya Medical Research Institute, Centre for Geographic Medicine Research (Coast), Kilifi, Kenya, Department of Paediatrics, Oxford University, Oxford, UK. and D.J. Senior Research Scientist Nokes, Kenya Medical Research Institute Centre for Geographic Medicine Research (Coast), Kilifi and Department of Biological Sciences, University of Warwick, Coventry, UK.

Request for reprints to: M. J. Ngama, Centre for Geographic Medicine Research (Coast), Kenya Medical Research Institute, Kilifi, Kenya

\title{
COMPARISON OF THREE METHODS OF COLLECTING NASAL SPECIMENS FOR RESPIRATORY VIRUS ANALYSIS
}

\author{
M. J. NGAMA, B. OUMA, M. E. ENGLISH and D. J. NOKES
}

\begin{abstract}
Background: Nasopharyngeal aspiration (NPA) is used widely in the collection of nasal specimens for respiratory virus diagnosis. The method has limitations in relation to technical expertise, patient anxiety, and apparatus dependence. Nasal washing (NW) offers an alternative approach.

Objective: To identify the merits of two different NW methods in comparison with NPA. Design: Two hundred children with acute respiratory infection (ARI) were randomised to receive one of three collection devices: (i) standard NPA, (ii) NW using a 30ml earsyringe bulb (NWb), or (iii) $\mathrm{NW}$ using a $5 \mathrm{ml}$ syringe $(\mathrm{NWs})$ with a shortened $(9 \mathrm{~cm})$ 8FG tube. Assessment focused on ease of procedure, acceptability to parent and child, and adequacy of epithelial cell yield for immunofluorescence testing. A short questionnaire was delivered.

Setting: Paediatric Ward of Kilifi District Hospital, (KDH) Kilifi, Kenya.

Subjects: Any child admitted with ARI between 5th November 2001 and 24th January 2002. Results: Children recruited into NPA, NWb and NWs procedures numbered 62, 76 and 62, respectively (median age of 8 months). A higher proportion of children receiving NWb did not cry $(43 \%)$ compared to those receiving NPA $(13 \%)$ (OR $5.18 ; 95 \%$ CI 2.17-12.4). Whereas $66 \%$ of mothers were comfortable with NPA procedure, the proportion for NWs was $40 \%$ (OR $0.341 ; 0.163-0.714)$. Acceptability to the operator was marginally lower for NWs than NPA (79\% vs 92\%, OR 0.324, 0.107-0.974). For other observations there were no differences between the procedures; these were length of procedure $(98 \%<5 \mathrm{mins})$, the acceptable time interval for repeating a procedure $(64 \%$ $<1$ week), comparison with blood collection (77\% preferred the nasal specimen) and slides with 20 or more epithelial cells (overall $82 \%$ ).

Conclusion: Nasal washing methods provide simple and effective alternatives to NPA, with the NWb being the more acceptable, and have merits for use in resource poor and home settings.
\end{abstract}

\section{INTRODUCTION}

Nasopharygeal aspiration (NPA) is the most common method used in health facilities to collect nasal specimens for respiratory virus diagnosis. The procedure requires technical competence for successful operation, has the potential to create anxiety in the patient and guardian, and is dependent upon mechanical or electrical suction apparatus for aspiration. These features are potential limitations for the application of NPA in settings where resources are minimal or when collection outside hospital is desired. An alternative approach of nasal washing (NW), in which collection fluid is instilled and aspirated from the nasal passage rather than dry aspiration, offers advantages over NPA for use both in the clinic and home settings. Here we report on a trial comparing two NW procedures(1,2) with NPA. Our aims were to identify the relative merits of each NW method compared to NPA in a rural district hospital, and, to a lesser degree, assess their merits for use in the home setting.

\section{MATERIALS AND METHODS}

Children with acute respiratory infection presenting to the paediatric ward of KDH between 5th November 2001 and 24th January 2002, entering a study of respiratory syncytial virus (KEMRI/National Ethical Review Committee SCC Protocol No. 594), were eligible for participation in this study. At informed consent, all the three devices were shown to the caretaker and the procedures explained, but only at the time of sampling was each child randomised to one of the procedures. The process of randomisation involved 
a dice labelled $\mathrm{A}, \mathrm{B}$ and $\mathrm{C}$ on each of two opposite sides (representing NPA, NWb and NWs, respectively). The dice was cast and the side facing up indicated the procedure for that participant.

Standard protocol was adopted for the collection of NPA samples. A 40cm 8FG tube (Suction tube, Pennine Healthcare, Derby, UK), attached to an electric pump (Laerdel suction unit, Laerdel Medical Products, Stavanger, Norway) via a trap (Mucus Specimen trap, Bard Ltd, UK) (Figure 1) was inserted gently into the nasopharynx through one nostril to a length equal to the distance from the nares to the tragus of the ear on the same side. Suction pressure $(200 \mathrm{mmHg})$ was applied and the catheter extracted whilst rotating (time taken for removal was no more than 10 seconds). Mucus was washed into the trap by aspirating $2 \mathrm{ml}$ of normal saline $(\mathrm{N} / \mathrm{s})$.

Two nasal washing methods were employed (Figure 1). The first, referred to as $\mathrm{NWb}$, employed a $30 \mathrm{ml}$ earsyringe bulb (Davol loz Ear Syringe, Abbott, Ashland, USA) to rapidly instill $5 \mathrm{ml}$ of $\mathrm{N} / \mathrm{s}$ into one nostril of the child (held upright with head slightly extended), followed by immediate aspiration of the fluid by releasing pressure on the bulb. Fluid emitted through the other nostril was collected into a $7 \mathrm{ml}$ bijou bottle, to which was added fluid from the bulb. If the resultant fluid was not cloudy (indicating the presence of mucus material), the procedure was repeated in the other nostril.

The second method, NWs, used a shortened (10 cm) 8FG tube (Ven Medical Products, Capetown, South Africa) attached to a $5 \mathrm{ml}$ syringe. The tube was inserted into the child's nasopharynx as for the NPA method, and $3 \mathrm{ml}$ of $\mathrm{N} / \mathrm{s}$ instilled, immediately sucked back into the syringe, and resultant fluid transferred to $7 \mathrm{ml}$ bijou. The child was laid on his/her side for the procedure.

Each procedure was carried out by the clinical officer (CO) overseeing the study $(\mathrm{MN})$ or by a ward field worker $(\mathrm{FW})$. Observations of the procedure and completion of a questionnaire were conducted by an assistant FW. Training of FWs (six in total) in the use of each collection device and the completion of the questionnaire occurred between 8th October 2001 and 4th November 2001 during which 50 samples were collected. Sample collection was overseen by the CO, who was also responsible for training, with support from the paediatrician (ME) and a video demonstration of the NWb method (kindly provided by Dr. P. Wright, Vanderbilt University Medical Center,USA).

A short questionnaire was formulated to quantify specific attributes of the procedures, in particular to assess the acceptability for nasal specimen collection. For each operation, the assistant FW recorded the (i) length of the procedure (from entry of mother/child into study room until exit), (ii) length of time the child cried once the procedure was initiated, (iii) mother's reaction to the procedure, and the responses to questioning on (iv) the length of time before the mother would allow a repetition of the procedure, and on (v) the acceptability of the procedure from the operator's point of view, ie ease of delivery, anxiety of the child's mother. We also asked the mother how the procedure compared with the collecting of a venous blood specimen (better/worse).

Samples collected were labelled and sent to the laboratory for processing within 30 minutes and stored at $+4^{\circ} \mathrm{C}$. Specimens were processed and slides produced within 48 hours of collection in preparation for immunofluorescent antibody testing (IFAT) for respiratory viruses. Slide preparation adopted standard manual cell separation up to 11th November 2001 and thereafter was facilitated by a cytology centrifuge (Cytospin 3, Thermo Shandon Ltd, Cheshire UK). For the latter, after breaking up mucus in the raw sample, $200 \mu 1$ of nasal washing or $150 \mu 1$ of NPA was loaded into a Cytofunnel, which was centrifuged at $1000 \mathrm{rpm}$ for 10 minutes in the Cytospin 3 to deposit cells onto a slide. Slides were air dried and assessed for adequacy by laboratory staff who recorded the number of epithelial cells observable on the preparation. Results were categorised as adequate ( $>20$ cells), present (1-19 cells), or absent.

Data were analysed using STATA V.7 (3). The Kruskal-Wallis rank test is used to test equality of age in procedure groups. We test for lack of independence in contingency table data using $X^{2}$ or Fisher's exact test. Logistic regression was used to compare $\mathrm{NWb}$ and NWs with NPA for each assessment variable (we first converted to binary outcomes), providing Odds Ratios (OR) with $95 \%$ confidence intervals.

\section{RESULTS}

Over the study period 200 children were enrolled of which 62, 76 and 62 were randomised to NPA, NWb and NWs procedures, respectively. The average age of all children was eight months (range 0-47), and 9 (042) for NPA, 6 (0-47) for NWb, and 11 (0-47) for NWs (Kruskal-Wallis (with ties) $\chi 2(2)=4.93, \mathrm{P}=0.085$ ). Results for the total sample are summarised as follows. In 194/ $199(97.5 \%)$ of cases the procedure took less than 5 minutes to perform. The child did not cry during 48/ $199(24.1 \%)$ of the procedures and in $2010.1 \%$ of cases the child cried for more than 1 minutes $(61.6 \%)$ 122/ 198 of mothers were judged comfortable with the procedure, with $8(4.0 \%)$ very disturbed, and (64.3\%) $126 / 196$ would allow the procedure to be repeated in one week, in contrast to $26(13.3 \%$ ) who said they would never want it to be repeated. For 171/199 (85.9\%) of the procedures the operator found the process easy and acceptable. In answer to the question comparing with blood collection, $145 / 189(76.7 \%)$ of the mothers said that the procedure of collecting a nasal specimen was better. In total, 164/200 (82.0\%) of the slides prepared were adequate ( $>20$ epithelial cells in a preparation) and in two (1.0\%) slides no epithelial cells were visible. Adoption of the cytospin method was not associated with a change in the proportion of slides being adequate compared to manual washing $82 \%(\mathrm{n}=185)$ vs $80 \%$ $(n=15)$, Fisher's exact 0.736 . 
Figure 1

Three nasal specimen collection devices. Nasal washing bulb (NWb), capacity 30ml (with 7ml bijou bottle), NPA apparatus consisting of $8 F G$ feeding tube attached to suction trap, with tube to pump (pump not shown), and nasal washing syringe (NWs), $5 \mathrm{ml}$, with attached short $(10 \mathrm{~cm}) 8 F G$ feeding tube

\section{Table 1}

Quantifiable comparisons between three methods of collecting nasal specimens

\begin{tabular}{|c|c|c|c|c|c|}
\hline Variable outcome & Method & $\begin{array}{l}\% \text { with } \\
\text { outcome }\end{array}$ & OR & $95 \% \mathrm{CI}$ & P-value \\
\hline \multirow[t]{3}{*}{ Did not cry } & NPA\# & 13 & 1 & & \\
\hline & $\mathrm{NWb}$ & 43 & 5.18 & $2.17-12.4$ & $<0.001$ \\
\hline & NWs & 11 & 0.875 & $0.296-2.58$ & 0.809 \\
\hline \multirow{4}{*}{$\begin{array}{l}\text { Mother } \\
\text { comfortable }\end{array}$} & & & & & \\
\hline & NPA & 66 & 1 & & \\
\hline & $\mathrm{NWb}$ & 75 & 1.54 & $0.733-3.22$ & 0.255 \\
\hline & NWs & 40 & 0.341 & $0.163-0.714$ & 0.004 \\
\hline \multirow{4}{*}{$\begin{array}{l}\text { Repeat after } \\
\text { one week }\end{array}$} & & & & & \\
\hline & NPA & 57 & 1 & & \\
\hline & $\mathrm{NWb}$ & 71 & 1.79 & $0.880-3.64$ & 0.108 \\
\hline & NWs & 63 & 1.28 & $0.618-2.66$ & 0.503 \\
\hline \multicolumn{6}{|l|}{ Acceptable } \\
\hline \multirow[t]{3}{*}{ to operator } & NPA & 92 & 1 & & \\
\hline & $\mathrm{NWb}$ & 87 & 0.579 & $0.187-1.79$ & 0.343 \\
\hline & NWs & 79 & 0.324 & $0.107-0.974$ & 0.045 \\
\hline \multirow{4}{*}{$\begin{array}{l}\text { Preferred to } \\
\text { blood collection }\end{array}$} & & & & & \\
\hline & NPA & 80 & 1 & & \\
\hline & $\mathrm{NWb}$ & 82 & 1.15 & $0.483-2.76$ & 0.748 \\
\hline & NWs & 66 & 0.487 & $0.210-1.13$ & 0.093 \\
\hline \multirow{4}{*}{$\begin{array}{l}\text { Adequate } \\
\text { cell number }\end{array}$} & & & & & \\
\hline & NPA & 79 & 1 & & \\
\hline & $\mathrm{NWb}$ & 88 & 1.97 & $0.782-4.99$ & 0.150 \\
\hline & NWs & 77 & 0.91 & $0.387-2.14$ & 0.828 \\
\hline
\end{tabular}

\#NPA, NWb, NWs. Nasopharyngeal aspirate, nasal washing bulb and nasal washing syringe 
Acceptability of procedures: Results of quantifiable comparisons between the three procedures using logistic regression are summarised in the Table 1. Age was excluded as a confounder since there was no difference in the age distribution between the groups. Only 5/199 procedures took longer than 5 minutes so no further analysis is presented. The NWb procedure was associated with significantly greater absence of crying in children than NPA (43\% versus 13\%). Mothers were observed to be less comfortable with NWs than NPA (40\% versus $66 \%$ ). There was no difference between methods in the mother's willingness to allow a repeat procedure. From the operator's perspective, NWs was marginally less acceptable than NPA (79\% versus $92 \%)$. Fewer mothers whose children had a sample collected by NWs thought this method was preferable to taking a blood specimen, when compared to mothers whose children received NPA, though this was not significant $(66 \%$ versus $80 \%$ ).

Adequacy of nasal specimens for IFAT: The proportion of samples from which adequate epithelial cells could be harvested did not differ significantly between collection devices (Table 1).

\section{DISCUSSION}

We undertook to compare the widely used method of nasopharyngeal aspiration with two nasal washing methods (NWb and NWs), for the collection of exfoliated epithelial cells for respiratory viral diagnosis.

A subjective assessment was made (by the CO) of the technical difficulty of each procedure, in relation to training and use in the current setting. The simpler equipment of the nasal washing alternatives compared to NPA does simplify training. However, each method has an associated technique that must be properly applied to ensure quality samples. Thus instruction, demonstration and assessment of use are required for all. In particular for the NWb, care is needed to ensure fluid reaches the nasopharynx. A sign of a successful $\mathrm{NWb}$ operation is emission of fluid from the other nostril. For both $\mathrm{NWb}$ and NWs procedures N/s can pass down the throat causing the child to gag. This problem appears more frequent with the NWs than $\mathrm{NWb}$ in our hands. In both NPA and NWs a feeding tube is inserted into the nasopharynx, and care must be taken not to cause trauma. Absence of the need for a mechanical/electrical pump suggests the alternative nasal washing methods might find more wide use in basic clinical facilities or in the home. However, limited availability of the shortened FG8 tube and the 30ml bulbs compared to standard FG8 catheters and suction traps may pose problems for general use.

Quantitative assessments identified the NWb to be more acceptable to the infant (less crying) than for NPA. Somewhat surprisingly, however, compared to NPA, not only was the NWs apparently less acceptable to the parent but also (marginally) the operator (the latter probably reflecting parental anxiety). No difference between the devices was observed in the length of procedure, the time before the mother would allow a repetition of the procedure, and comparability with blood collecting. Operator and parental assessments are highly subjective and should be viewed with some caution. The important issue is that however easy a technique may seem, a period of training is required for operators to become comfortable in its use, and time must be spent with the parent and child to put both at ease with the procedure.

No differences were observed between devices in adequacy of specimens for IFAT slide preparation. The measurement of numbers of epithelial cells is only an indication of the adequacy of the material for detecting viruses, and, in the case of the $\mathrm{NWb}$, since no part of the device is in physical contact with the nasopharynx, the source of cells is not being determined. Nevertheless, though not determined, it is likely that each device is collecting cells from a similar source in the nasal cavity.

Overall, this study indicates that nasal washing methods provide suitable alternatives to NPA, in terms of ease of use and equipment needs, and operationally, relative to NPA, the NWb has specific advantages in terms of acceptability. However, technical supervision is, at least initially, required for all methods, and attention is required to ensure acceptability to operator and recipient. Materials for nasal washing may currently be difficult to source, although this is likely to be resolved with an increased demand. Previous studies have demonstrated nasal washing to be operationally useful in the hospital $(1,4)$ and community $(5,6)$ settings, and have shown high viral titre yield(2,7). The procedure of nasal lavage, similar to NWs but with mechanical aspiration, has been shown to compare well with NPA in RSV diagnosis efficiency(8). Taken together with simpler equipment requirements, these facts suggest nasal washing methods may have considerable merit for use in minimal resource, and home, settings. In line with this, recent studies sponsored by the World Health Organisation to determine the community burden of RSV associated ARI follow recommendations to use nasal washing methods(9).

\section{ACKNOWLEDGEMENTS}

To the parents and their children who participated in the study, and field workers involved in collection of specimens. We acknowledge the support of Pat Cane, Birmingham University Medical School, UK, in establishing laboratory capacity, Shabir Madhi, University of Witwatersrand, RSA, for advice on use of the Cytospin 3 and the nasal washing syringe method, and Peter Wright, Vanderbilt University Medical Center, USA, for advice on the nasal washing bulb method. The study was supported by the Wellcome Trust (grant No: 061584). 


\section{REFERENCES}

1. Madhi, S.A., Schoub, B.D., Simmank, K., Blackburn, N., Klugman, K.P. Increased burden of respiratory viral associated severe lower respiratory tract infections in children infected with human immunodeficiency virus type 1. J. Pediat. 2000; 137: 78-84.

2. Hall, C. and Douglas, R.J. Clinically useful method for the isolation of respiratory syncytial virus. J. Infect. Dis. 1975; 131:1-5.

3. StataCorp. Stata Statistical Software: Release 7. 0: College Station, TX: Stata Corporation., 2001.

4. Wright, P.F., Gruber, W.C., Peters, M., et al. Illness severity, viral shedding, and antibody responses in infants hospitalized with bronchiolitis caused by respiratory syncytial virus. J. Infect. Dis. 2002; 185: 1011-1018.

5. Glezen, W., Paredes, A., Allison, J., Taber, L., and Frank, A.
Risk of respiratory syncytial virus infection for infants from low-income families in relationship to age, sex, ethnic group, and maternal antibody level. J. Pediat. 1981; 98:708-715.

6. Taber, L., Paredes, A., Glezen, W., and Couch, R. Infection with influenza A/Victoria virus in Houston families, 1976. J. Hyg. Cambridge. 1981; 86:303-313.

7. Hall, C., Douglas, G.J., and Geiman, J. Quantitative shedding patterns of respiratory syncytial virus in infants. J. Infec. Dis. 1975; 132:151-156.

8. Balfour-Lynn, I., Girdhar, D., and Aitken, C. Diagnosing respiratory syncytial virus by nasal lavage. Arch. Dis. Child. 1995; 72: 58-59.

9. Wright, P., and Cutts, F. Generic protocol to examine the incidence of lower respiratory infection (LRI) due respiratory syncytial virus (RSV) in children less than five years of age. Geneva: World Health Organization, 1996. 